\title{
Incorporating and evaluating citizen engagement in health research: a scoping review protocol
}

\author{
Anmol Shahid ${ }^{1}$, Brianna K. Rosgen ${ }^{1}$, Karla D. Krewulak', Diane L. Lorenzetti' ${ }^{2,3}$, Nadine Foster ${ }^{1}$, Bonnie G. Sept ${ }^{1}$, \\ Jeanna Parsons Leigh ${ }^{1,4}$, Henry T. Stelfox ${ }^{1,2,5}$ and Kirsten M. Fiest ${ }^{1,2,6^{*}}$
}

\begin{abstract}
Background: Citizen engagement in research is an emerging practice that involves members of the general public in research processes such as priority setting, planning, decision-making, research conduct, implementation, evaluation, and dissemination. Engaging citizens in research, particularly health research, increases the relevance of study findings, minimizes waste by facilitating stewardship over resources, and builds public trust in the research. While several existing frameworks guide the application of citizen engagement principles to health research, it is unclear how citizen engagement can be utilized to maximize benefits and minimize risks and challenges in health research. To address the gaps in knowledge around citizen engagement in health research, we propose a scoping review to synthesize the state of knowledge on methods to incorporate and evaluate citizen engagement in research. A protocol is presented in this manuscript.

Methods: The methodology for our scoping review is guided by Arksey and O' Malley's framework for scoping reviews, and additional recommendations by Levac and colleagues. We will include peer-reviewed and gray literature that report on citizen engagement in health research (including biomedical, clinical, health systems and services, and social, cultural, environmental and population health) and report method(s) to conduct, measure, or evaluate citizen engagement. We will systematically search electronic databases (MEDLINE, EMBASE, CINAHL, JSTOR, PsycINFO, Scopus, and Science Direct) from inception onwards and search relevant organizations' websites for additional studies, frameworks, and reports on citizen engagement. Title and abstract and full-text citations will be screened independently and in duplicate. Data will be extracted independently and in duplicate, including document characteristics, citizen engagement definitions and goals, and outcomes of citizen engagement (e.g., barriers, facilitators).
\end{abstract}

\footnotetext{
* Correspondence: kmfiest@ucalgary.ca

${ }^{1}$ Department of Critical Care Medicine, Cumming School of Medicine,

University of Calgary, Ground Floor, McCaig Tower, 3134 Hospital Drive NW, Calgary, Alberta T2N 5A1, Canada

${ }^{2}$ Department of Community Health Science and O'Brien Institute for Public

Health, Cumming School of Medicine, University of Calgary, Calgary, Alberta,

Canada

Full list of author information is available at the end of the article
}

(c) The Author(s). 2021 Open Access This article is licensed under a Creative Commons Attribution 4.0 International License, which permits use, sharing, adaptation, distribution and reproduction in any medium or format, as long as you give appropriate credit to the original author(s) and the source, provide a link to the Creative Commons licence, and indicate if changes were made. The images or other third party material in this article are included in the article's Creative Commons licence, unless indicated otherwise in a credit line to the material. If material is not included in the article's Creative Commons licence and your intended use is not permitted by statutory regulation or exceeds the permitted use, you will need to obtain permission directly from the copyright holder. To view a copy of this licence, visit http://creativecommons.org/licenses/by/4.0/ The Creative Commons Public Domain Dedication waiver (http://creativecommons.org/publicdomain/zero/1.0/) applies to the data made available in this article, unless otherwise stated in a credit line to the data. 
Discussion: This review will synthesize the definitions, goals, methods, outcomes, and significance of citizen engagement in health research, as well as any potential barriers, facilitators, and challenges outlined in existing literature. The findings will provide an evidence-based foundation for developing new or improved guidance for citizen engagement in health research. Overall, we anticipate that our scoping review will be a preliminary step to meaningful engagement of citizens in research and strengthen the relationship between the scientific community and the public through transparency and collaboration.

Systematic review registration: Open Science Framework https://osf.io/hzcbr.

Keywords: Citizen engagement, Public participation, Health research, Co-designed research, Models, Frameworks, Health services research, Scoping review, Protocol

\section{Background}

Citizen engagement in research is defined as the effective and systematic involvement of members of the general public or persons from affected community groups such as patients, caregivers, advocates, and representatives in research processes [1]. Organizations worldwide have suggested that citizen engagement in research may confer benefits to the conduct and uptake of scientific research through improving the relevance of study findings, encouraging the representation of diverse groups in research studies, minimizing waste by facilitating stewardship over resources, promoting mutual learning and understanding, and allowing broad dissemination of research findings beyond traditional academic audiences [2-6]. Most importantly, citizen engagement has demonstrated utility in building public trust in science and research [7]. This has led to citizen engagement becoming a priority for research funding organizations (e.g., the Canadian Institutes of Health Research [CIHR], National Institutes of Health $[\mathrm{NIH}]$ in the USA, and the National Institute for Health Research [NIHR] in the UK), policymakers, academic journals, and researchers themselves $[1,8-12]$. As a result, citizen engagement is now changing scientific research, particularly health research, to incorporate real-world lived-experiences and perspectives of members of the public.

Engaging citizens in research necessitates researchers to modify their research approach and critically evaluate their work to improve its generalizability and impact. However, while citizen engagement in research has been conceptually substantiated, it is unclear when, for whom, and how it can be most impactful [13]. Despite the availability of frameworks and guidelines for citizen engagement in health research $[1,2]$, there remains a lack of evidence-based methods to incorporate effective and systematic, or meaningful, engagement and valid measures to assess its impact.

Many studies intending to incorporate citizen engagement struggle to define citizen engagement, adequately describe processes used, discuss findings pertaining to citizen engagement, or share the impact of citizen engagement on their work [14]. To date, reviews and studies on citizen engagement in health research has largely focused on specific diseases, populations, and disciplines [15-20], limiting the generalizability of the findings. This highlights the need for a broad review and synthesis of citizen engagement in health research that could be used to develop a new framework of citizen engagement or enhance and refine existing frameworks with a strong evidentiary basis, ultimately leading to effective and unified citizen engagement strategies.

As the knowledge base on citizen engagement in health research is emerging, we will conduct a scoping review to identify and summarize literature on citizen engagement in health research. Specifically, we will conduct a scoping review to synthesize the state of knowledge on methods to incorporate and evaluate citizen engagement in this field. The outcomes of this scoping review will provide a descriptive summary of the literature on citizen engagement including frameworks, models, and interventions aimed at improving engagement in health research. This will yield foundational results including strengths and weaknesses in existing frameworks, models, and interventions, and help pinpoint areas that can be targeted to improve the relevance and effectiveness of future frameworks and recommendations.

\section{Methods}

This scoping review will be conducted according to recommendations specified by Arksey and O'Malley [21], and enhanced by Levac and colleagues [22]. The review protocol was registered within the Open Science Framework (https://doi.org/10.17605/OSF.IO/HZCBR) and is reported in accordance with the Preferred Reporting Items for Systematic Reviews and Meta-Analyses Protocols (PRISMA-P) statement [23] (checklist shown in Additional file 1). The proposed scoping review will be reported in accordance with the Preferred Reporting Items for Systematic Reviews and Meta-Analyses (PRISMA) extension for Scoping Reviews (PRISMA-ScR) [24]. 


\section{Stage 1: Identification of the research question}

We designed the research question to be broad in order to capture all potentially relevant information sources. This scoping review aims to answer the question: "what is the state of knowledge on methods to incorporate and evaluate citizen engagement in health research?" The proposed research question provides direction to the subsequent steps, and defines the scope of inquiry with respect to population, concept, and outcomes of interest [14].

For the purpose of this scoping review, our target population "citizens" will be defined as consumers of health services, informal caregivers, advocates, and representatives from community organizations, and members of the general public. Our target concept is engagement or participation in health research. For the purposes of this scoping review, we will adopt the CIHR definition of health research, which includes research in the biomedical, clinical, health systems and services, and social, cultural, environmental, and population health fields [18]. We recognize that engagement may take many forms, including but not limited to priority setting, planning, decision-making, research conduct, implementation, evaluation, or dissemination. Additionally, citizen engagement may occur when citizens are joint grant holders or co-applicants on research grants, help to identify research priorities, hold membership in a project advisory or steering group, comment and develop research materials, interact with research participants, and/or help carry out research activities.

\section{Stage 2: Identification of relevant studies}

We will identify relevant literature using a predetermined plan for data sources and search strategy, including search terms, languages, and dates of search. The search strategies will be designed to maximize the comprehensiveness and breadth of the search, while considering time and personnel workload as limiting factors $[14,15]$.

\section{Search strategy}

A search strategy will be developed by the study team, including a medical librarian (DLL), who has led multiple initiatives to develop, adapt, and evaluate approaches to incorporate citizen engagement into research [19-23]. The following databases will be searched from inception: MEDLINE (1879-present), EMBASE (1947-present), Cochrane Library (1996present), CINAHL (1961-present), PsycINFO (1927present), Scopus (1970-present), and Web of Science (1964-present). Our search will be broad to encompass relevant terminology in this area, including subject headings and keywords and relevant synonyms related to three concepts: (1) citizens (e.g., community member, lay person, public, stakeholder), (2) engagement (e.g., collaboration, engagement, participation), (3) health research (e.g., biomedical research, health research, public health research). No exclusion criteria will be placed on language, though we will exclude studies published prior to the year 2000. Prior to implementation, the search strategy will be independently reviewed using the Peer Review of Electronic Search Strategies (PRESS) checklist [24]. A draft search strategy (MEDLINE) is shown in Additional file 2.

A targeted search of the gray literature will also be conducted, searching relevant local, provincial, national, and international organizations' websites and related scientific or national funding organizations (i.e., OpenGrey, Trip, Involve, CIHR, Patient-Centered Outcomes Research Institute [PCORI]) for additional studies, frameworks, and reports on citizen engagement. Finally, the reference lists of included studies and related systematic reviews will also be screened to identify potentially relevant literature in health research.

\section{Stage 3: Study selection}

Following developing a strategy to identify studies, we will screen and select relevant studies for inclusion in the scoping review. As recommended by Levac and colleagues, we will develop inclusion and exclusion criteria a priori, with additional meetings with the study team to refine the study selection process at the beginning, midpoint, and endpoint of the citation screening process in case there are any unforeseen factors to be considered [15].

\section{Eligibility criteria}

Studies will be eligible for inclusion if they: (1) are primary (e.g., observational or interventional studies) or secondary (e.g., systematic or scoping reviews) research, frameworks, reviews, or reports; (2) report citizen engagement in health research (including biomedical, clinical, health systems and services, and social, cultural, environmental, and population health, as defined by CIHR); and (3) report method(s) to conduct, measure, or evaluate citizen engagement. No restrictions will be placed on language or study design. Studies published prior to the year 2000 will be excluded to capture a current reflection of citizen engagement in health research as citizen engagement is a research concept that has evolved significantly since its inception.

\section{Selection process}

Retrieved articles will be imported to Covidence (Veritas Health Innovation, Melbourne, Australia) for title and abstract screening, completed independently, and in duplicate by two reviewers. Reviewers will pilot screen the titles and abstracts of 50 articles to ensure consistency of 
inclusion and exclusion criteria and once a Kappa coefficient of inter-rater agreement $\geq 0.8$ is achieved, proceed to screen the remaining articles. If one reviewer indicates an article as potentially relevant at the title and abstract screening phase, the article will proceed to full-text review to ensure inclusivity. Following title and abstract screening, the full text of selected studies will be screened independently and in duplicate by two reviewers. Reviewers will pilot screen the full text of 20 articles, and proceed to screen the remaining articles once a Kappa coefficient of inter-rater agreement $\geq 0.8$ is achieved. Both reviewers must agree on inclusion status and reason for exclusion, if relevant. Any disagreements between the reviewers will be resolved by discussion or the involvement of a third reviewer, if required.

\section{Stage 4: Charting the data}

We will chart the data beginning with defining the information to be extracted from the studies by developing an initial data charting form and then refining this form iteratively and through regular discussion with the study team. The study team will develop a standardized data extraction form in Microsoft Excel (version 16.29.1). The data extraction form will be piloted by two reviewers with ten included studies, and revised as needed. Once the final data abstraction form is developed, all relevant articles will then be abstracted independently and in duplicate by two reviewers.
Extracted variables in the data extraction form will include (1) citation details (i.e., author(s), year, country, publisher, document/ research type, (2) participant details (i.e., population of focus, age, sex, gender, geographical location), (3) citizen engagement operationalization (e.g., definitions, type, goals of citizen engagement), (4) study characteristics (i.e., design, sample size, outcome measurement), (5) findings and outcomes of citizen engagement, and (6) any benefits, limitations, challenges, and risks of citizen engagement in the context of health research. A detailed list of variables to be extracted is shown in Table 1.

Stage 5: Collating summarizing and reporting the results Following collation and analysis of the results [21, 22], we will report (i) a PRISMA-ScR checklist to guide reporting and the flow diagram to report the number of unique articles identified, excluded articles, reasons for exclusion, and included articles in the final scoping review [24]; (ii) citation and participant details using descriptive statistics and accompanying interval estimates; (iii) using tables for key characteristics of studies included study findings, methods of citizen engagement presented, and barriers, facilitators, and knowledge gaps. We will accompany the tables with a narrative synthesis of extracted data. The narrative synthesis will also present any limitations of studies included, knowledge gaps identified, and highlight areas that need further research.

Table 1 Draft list of variables to be extracted

\begin{tabular}{|c|c|c|}
\hline Citation details & \multicolumn{2}{|c|}{$\begin{array}{l}\text { List of authors (surnames) } \\
\text { Year of publication } \\
\text { Country } \\
\text { Publisher (e.g., journal, institution) } \\
\text { Document type (e.g., framework OR model OR report) } \\
\text { Type of research (i.e., primary study OR secondary study) } \\
\text { Research area (e.g., health sciences, social sciences, and natural sciences and engineering) }\end{array}$} \\
\hline $\begin{array}{l}\text { Characteristics of intended } \\
\text { participants (i.e., citizens) }\end{array}$ & \multicolumn{2}{|c|}{$\begin{array}{l}\text { Population of focus (patient, family, healthcare provider, researcher, knowledge-user, decision-maker) } \\
\text { Age } \\
\text { Sex } \\
\text { Gender } \\
\text { Geographical location }\end{array}$} \\
\hline \multirow[t]{3}{*}{ Citizen engagement } & Operationalization & $\begin{array}{l}\text { Definition of citizen engagement } \\
\text { Type and goals of engagement (e.g., priority setting, decision-making, research con- } \\
\text { duct, implementation, evaluation, dissemination) } \\
\text { Engagement frameworks used } \\
\text { Engagement uptake }\end{array}$ \\
\hline & Methods (if applicable) & $\begin{array}{l}\text { Study design } \\
\text { Sample size } \\
\text { Outcome measurement }\end{array}$ \\
\hline & $\begin{array}{l}\text { Results and discussion } \\
\text { (if applicable) }\end{array}$ & $\begin{array}{l}\text { Findings and outcomes of citizen engagement } \\
\text { Barriers to citizen engagement } \\
\text { Facilitators of citizen engagement } \\
\text { Benefits of citizen engagement } \\
\text { Challenges and risks of citizen engagement } \\
\text { Strategies to mitigate challenges and risks of citizen engagement } \\
\text { Author-stated conclusions (if applicable) }\end{array}$ \\
\hline
\end{tabular}




\section{Stage 6: Consultation}

We will involve citizens (NF, BS) in study conception and design as well as interpretation and contextualization of the data. This stakeholder consultation (i.e., citizen engagement) will allow for the incorporation of perspectives and insights beyond the literature and is necessary to improve the rigor and uptake of findings $[14,15]$.

\section{Discussion}

This scoping review aims to synthesize published research, guidelines, and frameworks relevant to citizen engagement in health research. The findings of this scoping review will be applicable to many research disciplines beyond health research such as social sciences research and natural sciences research. The outcome of this scoping review will be a summary of the definitions, goals, methods, outcomes, significance, and evaluation of citizen engagement alongside potential barriers, facilitators, benefits, challenges, or risks.

The potential findings of this scoping review will have several applications. First, we will provide an evidencebased foundation to create novel guidance for citizen engagement in research or enhance existing guidelines, such as those developed by CIHR [1] and NIHR [1, 3]. Second, this scoping review may highlight potential gaps in the literature, directing future areas of study in the science of citizen engagement in health research. Third, our scoping review will act as a comprehensive information source of citizen engagement in research that research teams may learn from and apply to their own work. Fourth, this scoping review will identify where sufficient literature exists to warrant systematic reviews that could inform citizens of their potential to be impactful co-developers of research across many disciplines. Overall, we hope that our study will be a preliminary step to meaningful and effective engagement of citizens in research to improve the impact and uptake of research findings, and strengthen the relationship between the scientific community and the public through transparency and collaboration.

This scoping review will have some limitations. First, due to lack of standardization in terminology and definitions of citizen engagement [25], we may not capture all literature pertaining to the conduct, measurement, or evaluation of citizen engagement. To ensure we capture most relevant literature, we have developed a search strategy with a medical librarian experienced in literature reviews in the health sciences. Additionally, due to intensive NIHR efforts to set standards for patient and public involvement (i.e., citizen engagement) [26], much of the literature on citizen engagement in health research originates from the UK $[25,27-29]$. Consequently, our scoping review will likely include more UK- based citizen engagement literature than any other country. To ensure we do not misrepresent worldwide citizen engagement practices, we will clearly state the geographical location of any included studies and highlight any practices distinct to the UK.

\section{Team preparedness and dissemination of research findings}

We are well-positioned to complete this project due to our knowledge and experience in engaging and evaluating the impact of citizen engagement in health research. We have led initiatives to develop, adapt, and evaluate approaches to incorporate citizen engagement into research and routine clinical care through our research programs [19-23]. We have experience with engaging and training patient and family partners in critical care medicine research (e.g., generating ideas, writing grants, priority setting, conducting research, writing manuscripts), and involving patient advisors in communication of research findings to members of the public at open engagement sessions [19-23]. Additionally, we are well-equipped (i.e., large multidisciplinary team comprised of trainees, staff, and senior researchers) to undertake the vast literature this scoping review will capture. We have a track record of high-quality and pertinent systematic and scoping review publications that inform our research [30-33]. Given that data is publicly available (i.e., published articles and online material), we do not anticipate any data-sharing issues in this work.

Any amendments made to this protocol when conducting the study will be outlined in the Open Science Framework protocol and reported in the final manuscript. The findings of this scoping review will be disseminated widely by the study team members through our research team and institutional websites and presentations at local, national, and international conferences. Our citizen research partners will aid in disseminating the study findings (both to lay and scientific audiences), a step we consider critical for this work is to have an impact on citizen engagement in research. We intend to submit the findings for peerreviewed publication.

\section{Abbreviations}

CIHR: Canadian institutes of health research; NIH: National institutes of health; NIHR: National institute for health research; PRESS: Peer review of electronic search strategies; PRISMA-SCR: Preferred reporting items for systematic reviews and meta-analyses extension for scoping reviews

\section{Supplementary Information}

The online version contains supplementary material available at https://doi. org/10.1186/s13643-021-01812-4.

Additional file 1:. PRISMA-P 2015 Checklist

Additional file 2:. Medline Search Strategy 


\section{Acknowledgements}

Not applicable.

\section{Authors' contributions}

All authors (AS, BKR, KDK, DLL, JPL, NF, BS, HTS, and KF) have contributed to the conception and design of this protocol. AS and BKR drafted the manuscript. All authors critically revised the manuscript and approved of the final submitted version.

\section{Authors' information}

KMF is the Director of Research and Innovation for the Department of Critical Care Medicine and an assistant professor in the Departments of Critical Care Medicine, Community Health Sciences, and Psychiatry at the University of Calgary. HTS is the scientific director of the O'Brien Institute, a member of the Canadian Academy of Health Sciences, and a professor in the Department of Critical Care Medicine at the University of Calgary. JPL is an assistant professor in the Department of Critical Care Medicine at Dalhousie University and an adjunct professor in the Department of Critical Care Medicine at the University of Calgary.

\section{Funding}

This study is currently unfunded but is seeking local and national sources of funding.

Availability of data and materials

Not applicable.

\section{Declarations}

Ethics approval and consent to participate

Not applicable.

\section{Consent for publication}

Not applicable.

\section{Competing interests}

The authors declare that they have no competing interests.

\section{Author details}

'Department of Critical Care Medicine, Cumming School of Medicine, University of Calgary, Ground Floor, McCaig Tower, 3134 Hospital Drive NW Calgary, Alberta T2N 5A1, Canada. ${ }^{2}$ Department of Community Health Science and O'Brien Institute for Public Health, Cumming School of Medicine, University of Calgary, Calgary, Alberta, Canada. ${ }^{3}$ Health Sciences Library, University of Calgary, Calgary, Alberta, Canada. ${ }^{4}$ Dalhousie University, Halifax, Nova Scotia, Canada. ${ }^{5}$ Alberta Health Services, Calgary, Alberta, Canada. ${ }^{6}$ Department of Psychiatry and Hotchkiss Brain Institute, Cumming School of Medicine, University of Calgary, Calgary, Alberta, Canada.

\section{Received: 1 February 2021 Accepted: 10 September 2021}

\section{Published online: 28 September 2021}

\section{References}

1. Canadian Institutes of Health Research. CIHR's framework for citizen engagement. Ottawa, ON; 2012.

2. INVOLVE. Exploring impact: public involvement in NHS, public health and social care research. 2009.

3. National Institute for Health Research (NIHR). Going the extra mile: Improving the nation's health and wellbeing through public involvement in research. London. 2014. [Available from: https://www.nihr.ac.uk/ documents/about-us/our-contribution-to-research/how-we-involve-patientscarers-and-the-public/Going-the-Extra-Mile.pdf].

4. International Association for Public Participation. Core Values for the Practice of Public Participation Sooke, BC: Internal Canada; 2018 [Available from: https://www.iap2canada.ca/foundations]

5. Brett J, Staniszewska S, Mockford C, Herron-Marx S, Hughes J, Tysall C, et al. A systematic review of the impact of patient and public involvement on service users, researchers and communities. Patient. 2014;7(4):387-95. https://doi.org/10.1007/s40271-014-0065-0.

6. Shimmin C, Wittmeier KDM, Lavoie JG, Wicklund ED, Sibley KM. Moving towards a more inclusive patient and public involvement in health research paradigm: the incorporation of a trauma-informed intersectional analysis. BMC Health Serv Res. 2017;17(1):539. https://doi.org/10.1186/s12913-017-2463-1.

7. Haywood BK. Beyond data points and research contributions: the personal meaning and value associated with public participation in scientific research. International Journal of Science Education, Part B. 2016;6(3):23962. https://doi.org/10.1080/21548455.2015.1043659.

8. Walshe K, McKee M, Groenewegen P, Hansen J, Figueras J, Boccia S, et al. Reshaping the agenda of the European Commission for the health systems and policy research in Europe within Horizon 2020. Epidemiol Biostat Public Health. 2013;10(9893):668-9. https://doi.org/10.1016/S0140-6736(12)62195-3.

9. Horobin A. Going the extra mile - creating a co-operative model for supporting patient and public involvement in research. Res Involv Engagem. 2016;2:9.

10. Price A, Schroter S, Snow R, Hicks M, Harmston R, Staniszewska S, et al. Frequency of reporting on patient and public involvement (PPI) in research studies published in a general medical journal: a descriptive study. BMJ Open. 2018;8(3):e020452. https://doi.org/10.1136/bmjopen-2017-020452.

11. Social Sciences and Humanities Research Council. Community engagement 2019 [Available from: https://www.sshrc-crsh.gc.ca/society-societe/ community-communite/index-eng.aspx.

12. Natural Sciences and Engineering Research Council of Canada. NSERC 2020 A strategic plan 2020 [June 23 2020]. Available from: https://www.nserccrsng.gc.ca/nserc-crsng/nserc2020-crsng2020/index eng.asp.

13. Marris $C$, Rose N. Open engagement: exploring public participation in the biosciences. PLoS Biol. 2010;8(11):e1000549. https://doi.org/10.1371/journal. pbio.1000549.

14. Arksey H, O'Malley L. Scoping studies: towards a methodological framework Int J Soc Res Methodol. 2005;8(1):19-32. https://doi.org/10.1080/1364557032 000119616.

15. Levac D, Colquhoun H, O'Brien KK. Scoping studies: advancing the methodology. Implementation Sci. 2010;5(1):69. https://doi.org/10.1186/174 8-5908-5-69.

16. Shamseer L, Moher D, Clarke M, Ghersi D, Liberati A, Petticrew M, et al. Preferred reporting items for systematic review and meta-analysis protocols (PRISMA-P) 2015: elaboration and explanation. BMJ. 2015;350(jan02 1):g7647. https://doi.org/10.1136/bmj.g7647.

17. Tricco AC, Lillie E, Zarin W, O'Brien KK, Colquhoun H, Levac D, et al. PRISMA Extension for Scoping Reviews (PRISMA-SCR): checklist and explanation. Ann Intern Med. 2018;169(7):467-73. https://doi.org/10.7326/M18-0850.

18. Research $\mathrm{CloH}$. ClHR background: Government of Canada; 2019 [Available from: https://letstalk-cihr.ca/about.

19. Fiest KM, Krewulak KD, Ely EW, Davidson JE, Ismail Z, Sept BG, et al. Partnering with family members to detect delirium in critically ill patients. Crit Care Med. 2020;48(7):954-61. https://doi.org/10.1097/CCM. 0000000000004367

20. Fiest KM, Krewulak KD, Sept BG, Spence KL, Davidson JE, Ely EW, et al. A study protocol for a randomized controlled trial of family-partnered delirium prevention, detection, and management in critically ill adults: the ACTIVATE study. BMC Health Serv Res. 2020;20(1):453. https:/doi.org/10.1186/s12913-020-05281-8.

21. Krewulak KD, Sept BG, Stelfox HT, Ely EW, Davidson JE, Ismail Z, et al. Feasibility and acceptability of family administration of delirium detection tools in the intensive care unit: a patient-oriented pilot study. CMAJ Open. 2019:7(2):E294-e9. https://doi.org/10.9778/cmajo.20180123.

22. Boyd JM, Burton R, Butler BL, Dyer D, Evans DC, Felteau M, et al. Development and validation of quality criteria for providing patient- and family-centered injury care. Ann Surg. 2017;266(2):287-96. https://doi.org/1 0.1097/SLA.0000000000002006.

23. Potestio ML, Boyd JM, Bagshaw SM, Heyland D, Oxland P, Doig CJ, et al. Engaging the public to identify opportunities to improve critical care: a qualitative analysis of an open community forum. PLoS One. 2015;10(11): e0143088. https://doi.org/10.1371/journal.pone.0143088.

24. McGowan J, Sampson M, Salzwedel DM, Cogo E, Foerster V, Lefebvre C. PRESS peer review of electronic search strategies: 2015 guideline statement. J Clin Epidemiol. 2016;75:40-6. https://doi.org/10.1016/j.jclinepi.2016.01.021.

25. Miah J, Dawes P, Edwards S, Leroi I, Starling B, Parsons S. Patient and public involvement in dementia research in the European Union: a scoping review. BMC Geriatr. 2019;19(1):220. https://doi.org/10.1186/s12877-019-1217-9.

26. Greenhalgh T, Hinton L, Finlay T, Macfarlane A, Fahy N, Clyde B, et al. Frameworks for supporting patient and public involvement in research: systematic review and co-design pilot. Health Expect. 2019:22(4):785-801. https://doi.org/10.1111/hex.12888 
27. Modigh A, Sampaio F, Moberg L, Fredriksson M. The impact of patient and public involvement in health research versus healthcare: a scoping review of reviews. Health Policy. 2021;125(9):1208-21. https://doi.org/10.1016/j.hea Ithpol.2021.07.008.

28. Flynn $R$, Walton S, Scott SD. Engaging children and families in pediatric health research: a scoping review. Res Involv Engagem. 2019;5(1):32. https:// doi.org/10.1186/s40900-019-0168-9.

29. Bird M, Ouellette C, Whitmore C, Li L, Nair K, McGillion MH, et al. Preparing for patient partnership: a scoping review of patient partner engagement and evaluation in research. Health Expect. 2020;23(3):523-39. https://doi. org/10.1111/hex.13040

30. Bowen DJ, Kreuter M, Spring B, Cofta-Woerpel L, Linnan L, Weiner D, et al. How we design feasibility studies. Am J Prev Med. 2009;36(5):452-7. https:// doi.org/10.1016/j.amepre.2009.02.002.

31. Cherak SJ, Fiest KM, VanderSluis L, Basualdo-Hammond C, Lorenzetti DL, Buhler $S$, et al. Nutrition interventions in populations with mental health conditions: a scoping review. Appl Physiol Nutr Metab. 2020;45(7):687-97. https://doi.org/10.1139/apnm-2019-0683.

32. Rosgen B, Krewulak K, Demiantschuk D, Ely EW, Davidson JE, Stelfox HT, et al. Validation of caregiver-centered delirium detection tools: a systematic review. Journal of the American Geriatrics Society. 2018;66(6):1218-25. https://doi.org/10.1111/jgs.15362

33. Cherak SJ, Rosgen BK, Amarbayan M, Plotnikoff K, Wollny K, Stelfox HT, et al. Impact of social media interventions and tools among informal caregivers of critically ill patients after patient admission to the intensive care unit: a scoping review. PLoS One. 2020;15(9):e0238803. https://doi.org/10.1371/ journal.pone.0238803.

\section{Publisher's Note}

Springer Nature remains neutral with regard to jurisdictional claims in published maps and institutional affiliations.

Ready to submit your research? Choose BMC and benefit from:

- fast, convenient online submission

- thorough peer review by experienced researchers in your field

- rapid publication on acceptance

- support for research data, including large and complex data types

- gold Open Access which fosters wider collaboration and increased citations

- maximum visibility for your research: over $100 \mathrm{M}$ website views per year

At BMC, research is always in progress.

Learn more biomedcentral.com/submissions 